\title{
Quasielastic Light Scattering for Protein Assembly Studies
}

\author{
Aleksey Lomakin, David B. Teplow, and George B. Benedek
}

Summary

Quasielastic light scattering (QLS) spectroscopy is an optical method for the determination of diffusion coefficients of particles in solution. In this chapter, we discuss the principles and practice of QLS with respect to protein assembly reactions. Particles undergoing Brownian motion produce fluctuations in scattered light intensity. We describe how the temporal correlation function of these fluctuations can be measured and how this correlation function provides information about the distribution of diffusion coefficients of the particles in solution. We discuss the intricacies of deconvolution of the correlation function and the assumptions incorporated into data analysis procedures. We explain how the Stokes-Einstein relationship can be used to convert distributions of diffusion coefficients into distributions of particle size. Noninvasive observation of the temporal evolution of particles sizes provides a powerful tool for studying protein aggregation and self-assembly. We use examples from studies of $A \beta$ fibrillogenesis to illustrate QLS application for understanding the molecular mechanisms of the nucleation and growth of amyloid fibrils.

Key Words: Dynamic light scattering; diffusion; size distribution; self-assembly; aggregation; Alzheimer's disease, amyloid; fibrillogenesis.

\section{Introduction}

Quasielastic light scattering (QLS), also known as dynamic light scattering (DLS), is an optical method well-suited for the determination of diffusion coefficients of particles undergoing Brownian motion in solution (1,2). Diffusion coefficients are determined by particle size, shape, and flexibility, as well as by inter-particle interactions. All these parameters provide important information about the kinetics and structural transitions within systems of particles in solution and can be studied by QLS. The QLS method is rapid, sensitive, non-invasive, and quantitative. QLS instruments may be constructed relatively easily and are also available commercially.

From: Methods in Molecular Biology, vol. 299: Amyloid Proteins: Methods and Protocols Edited by: E. M. Sigurdsson $\odot$ Humana Press Inc., Totowa, NJ 
QLS is a useful tool for studying particle aggregation and, in particular, for monitoring protein assembly. The processes of protein assembly are ubiquitous. They are required to produce multi-subunit structures, including enzymes, enzyme complexes, ribosomes, ion channels, and viral capsids. Protein assembly reactions also are associated with a number of diseases, including Alzheimer's (3), prion (4), and other neurodegenerative diseases (5), sickle cell anemia (6), and cataract disease (7). In each case, proteins that exist normally in a soluble, disaggregated state assemble into oligomeric and polymeric structures which cause cell and tissue injury, disrupting normal organismal function. To study the factors controlling both normal and pathological protein assembly processes, a method is required to monitor these processes with high sensitivity and resolution. The subject of this chapter, QLS, is such a method (8).

In its essence, QLS simply measures the fluctuations in light intensity emanating from a sample irradiated by a laser. These fluctuations contain information about the physical nature of the particles in the sample solution. However, for this information to be interpreted accurately, the theoretical and practical underpinnings of the method must be understood and incorporated into the execution of the QLS experiment. To assist the reader in this endeavor, this chapter is organized in a tripartite manner. The introduction to Subheading 2. provides a general overview of the entire QLS method. The remainder of Subheading 2. reviews general principles of light scattering, including the critical mathematical formulations upon which the method is based. Subheading 3. discusses the QLS hardware, practical aspects of its use, and how the raw data are analyzed to produce a physical picture of the particles in the solution under study. Accompanying these sections are illustrative examples of QLS in practice.

\section{QLS Theory}

Coherent light generated by a laser is an electromagnetic wave. Particles irradiated by this wave produce secondary waves, i.e., scattered light. This scattered light has a number of characteristics, which make its analysis of experimental interest. The amplitude of the scattered wave depends on scatterer mass and refractive index. The phase of the scattered wave depends on the position of the scattering particle. The intensity of the scattered light is the square of the sum of amplitudes of all scattering waves with their phases taken into account (see Subheading 2.1.). As the scattering particles move, the phases of their scattered electromagnetic waves change, causing fluctuations in the intensity of light registered by a photodetector. The essence of the QLS technique is the measurement of the temporal correlations in the fluctuations in the scattered light intensity (see Subheading 2.2.), and from analysis of these data, the reconstruction the dynamics of scatterer motion. 
The QLS method probes dynamics of the scattering system on a time scale ranging from tens of nanoseconds to seconds. The dynamics, which is of a particular interest for studying protein aggregation, is the diffusive motion of the particles in solution. The measurement of the intensity correlation function allows evaluation of the diffusion coefficients of the scattering particles (see Subheading 2.3.). In studies of protein aggregation, the sample usually contains particles of different sizes. In these polydisperse systems, "regularization" procedures provide the means to reconstruct smooth distributions of the scattering intensity over the scatterers' diffusion coefficient (see Subheading 3.3.).

The diffusion coefficient depends on particle size and shape, as well as on the ambient temperature and solution viscosity. By converting a diffusion coefficient into a hydrodynamic radius (the radius of a hard sphere with the same diffusion coefficient as the scatterer), temperature and viscosity are factored out (see Subheading 3.4.1.). The intensity of scattering by an individual particle is proportional to its mass squared (see Subheading 2.1.2.). This makes the QLS method particularly sensitive to large scatterers. If the relationship between particle mass and size is known, the distribution of the relative concentrations of particles over their size can be reconstructed (see Subheading 3.4.3.).

A QLS instrument consists of a laser with beam delivery optics, a cuvet containing the sample, a photodetector with light-collecting optics, and a correlator with data analysis software (see Subheading 3.1.). The degree to which the user has control over instrument settings varies from system to system. The more expensive systems usually allow more user control and require more expertise. Here, we focus on key general features of QLS method, features whose proper use and optimization are critical for the successful implementation of the QLS method.

\subsection{General Principles of Light Scattering}

\subsubsection{Scattering Vector}

Relative to the phase of a wave scattered at the origin, the phase of a wave scattered at a point with radius vector $\mathbf{r}$ is $\mathbf{q} \cdot \mathbf{r}(\mathbf{F i g}$. 1). The vector $\mathbf{q}$ is called the "scattering vector" and is a fundamental characteristic of any scattering process. Its length is $q \equiv|\mathbf{q}|=4 \pi / \lambda \sin \theta / 2$, where $\theta$ is the scattering angle and $\lambda$ is the light wavelength in the scattering medium, $\lambda=\lambda_{0} / n$. Here, $n$ is the refractive index of the medium and $\lambda_{0}$ is the wavelength of the incident light in vacuum. The summary electromagnetic field resulting from scattering by many particles is

$$
\mathrm{E}=\sum_{k} \mathbf{E}_{k} \exp \left(i \mathbf{q} \cdot \mathbf{r}_{k}+i \varphi\right),
$$

where $\mathbf{E}_{k}$ is the amplitude of the wave scattered by the $k$-th particle located at position $\mathbf{r}_{k}$, and $\varphi$ is the common phase shift equal to the phase of the putative 


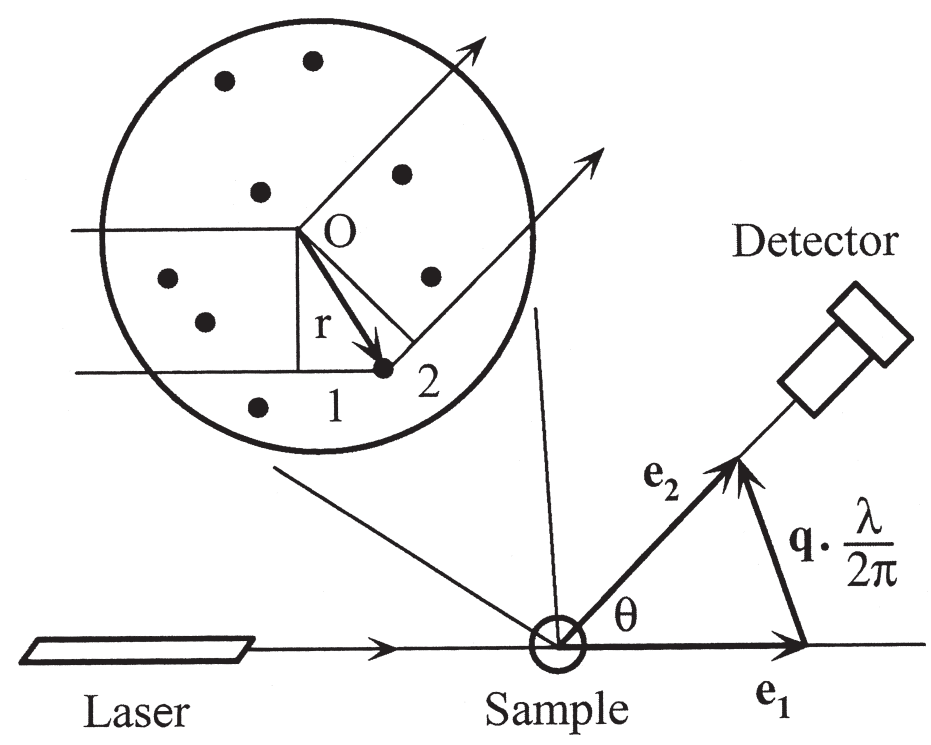

Fig. 1. The scattering vector $\mathbf{q}$. The path traveled by a wave scattered at the point with radius vector $\mathbf{r}$ differs from the path passing through the reference point $\mathrm{O}$ by two segments, 1 and 2, with lengths $l_{1}$ and $l_{2}$, respectively. The phase difference is $\Delta \phi=2 \pi$ $\left(l_{1}+l_{2}\right) / \lambda$. The segment $l_{1}$ is a projection of $\mathbf{r}$ on a unit vector $\mathbf{e}_{1}$ in the direction of the incident beam, i.e., $l_{1}=\mathbf{r} \cdot \mathbf{e}_{1}$. Similarly, $l_{2}=\mathbf{r} \cdot \mathbf{e}_{2}$, where $\mathbf{e}_{2}$ is a unit vector in the direction of scattering. Thus $\Delta \phi=(2 \pi / \lambda)\left(\mathbf{e}_{1}-\mathbf{e}_{2}\right) \cdot \mathbf{r}$. The vector $\mathbf{q}=(2 \pi / \lambda)\left(\mathbf{e}_{1}-\mathbf{e}_{2}\right)$ is called the scattering vector.

wave scattered at the origin, $\mathbf{r}=0$. The intensity $I$ of the scattered light per unit area is proportional to the square of the amplitude of the electromagnetic field, that is

$$
I \propto|\mathbf{E}|^{2}=\left|\sum_{k} \mathbf{E}_{k} \exp \left(i \mathbf{q} \cdot \mathbf{r}_{k}\right)\right|^{2}
$$

\subsubsection{Intensity of Scattering by a Small Particle}

Consider an aggregate composed of $m$ monomers, each producing scattered electromagnetic wave $\mathbf{E}_{0}$. Let the aggregate center of mass be at point $\mathbf{R}$. If the size of the aggregate is small, so that $\mathbf{q} \cdot\left(\mathbf{r}_{k}-\mathbf{R}\right)<<1$ for all monomers, Eq. 1 reduces to $\mathbf{E} \approx m \mathbf{E}_{0} \exp (i \mathbf{q} \cdot \mathbf{R}+i \varphi)$. Thus the intensity of the light scattered by the aggregate is proportional to the aggregation number squared, $I=m^{2} I_{0}$, where $I_{0}$ is the intensity of scattering by a monomer. The quadratic dependency of scattering intensity on the mass of the scatterer is the basis for optical determination of the molecular weight of macromolecules (9), for various turbidimetry and nephelometry techniques, and for understanding many natural phenomena, from appearance of clouds to cataractogenesis in the eye lens (10). 


\subsubsection{Intensity of Scattering by a Large Particle}

If an aggregate particle is not small, the destructive interference of waves scattered from different points in the particle must be taken into account. This destructive interference reduces the intensity of light scattering by a factor of $|\alpha|^{2}$, where $\alpha(\mathbf{q})$ is an averaged value of the phase factors $\exp \left(i \mathbf{q} \cdot \mathbf{r}_{\mathrm{k}}\right)$ or all monomers (see Note 1). The factor $|\alpha|^{2}$ should be averaged over all possible orientations of the particle. The result of this averaging yields the structure factor. A table of expressions for the structure factors for particles of various shapes can be found elsewhere (11).

\subsubsection{Intensity of Light Scattered by Many Particles}

In a typical experiment, light scattered by $N$ individual molecules at random locations $\mathbf{r}_{k}$ within the scattering volume is detected. The size of the scattering volume generally is large, thus $\mathbf{q} \cdot \mathbf{r}_{k} \gg 1$ and the phase factors $\exp \left(i \mathbf{q} \cdot \mathbf{r}_{k}\right)$ in Eq. 1 vary randomly. As a result, the mean square amplitude of the scattered wave is proportional to $\sqrt{N}$, and the average intensity of the scattered light is simply $N$ times the intensity scattered by an individual particle, as expected. The local intensity, however, fluctuates from one point to another around its average value, as described by Eq. 2 . The pattern of these fluctuations in light intensity, called an interference pattern or "speckles," is determined by the positions of the scattering particles. The characteristic size of the speckles is called coherence length.

\subsection{Correlation Function}

\subsubsection{Correlation Function of Intensity Fluctuations}

In QLS, the photodetector registers the fluctuations of the intensity of the scattered light, which are in fact random. Information is contained only in the temporal correlations in this signal. The correlation function of the intensity $I(t)$ is defined as:

$$
G^{(2)}(\tau)=\langle I(t) I(t+\tau)>.
$$

In the above formula, the angular brackets denote an average over time $t$. This time averaging, an inherent feature of the QLS method, is necessary to extract information from the random fluctuations in the intensity of the scattered light.

\subsubsection{Connection to Field Correlation Function}

The notation $G^{(2)}(\tau)$ had been introduced to distinguish the correlation function of the intensity $I(t)$ from the correlation function of the electromagnetic field

$$
G^{(1)}(\tau)=<\mathbf{E}(t) \mathbf{E}^{*}(t+\tau)>,
$$


which is the Fourier transform of the light spectrum. In the majority of practical applications of QLS, the scattered light is a sum of waves scattered by many independent particles and therefore displays Gaussian statistics. This being the case, there is a relation between the intensity correlation function $G^{(2)}(\tau)$ and the field correlation function $G^{(1)}(\tau)$ :

$$
G^{(2)}(\tau)=I_{0}^{2}\left(1+\gamma g^{(1)}(\tau)^{2}\right) .
$$

Here $I_{0}$ is the average intensity of the detected light and $\gamma$ is the efficiency factor. When scattered light is collected from a small area, $\gamma \approx 1$. If light is collected from area large compared to the coherence area, fluctuations in light intensity are averaged out and $\gamma<<1$. The key element in Eq. 5 is $g^{(1)}(\tau)=\mid G^{(1)}(\tau) / G^{(1)}$ (0) |, the absolute value of the normalized field correlation function. This is the instrument independent function that is measured and analyzed in the QLS method.

\subsection{Dynamics of the Scattering System}

\subsubsection{Brownian Motion}

The dynamics which is of particular interest for studying protein aggregation is Brownian motion of the particles. Brownian motion is responsible for the diffusion of the solute and is quantitatively characterized by the diffusion coefficient $D$. According to Eq. 1, electromagnetic waves scattered by a pair of individual particles have, at the observation point, a phase difference of $\mathbf{q} \cdot \Delta \mathbf{r}$, where $\Delta \mathbf{r}$ is the vector distance between particles. As the scattering particles move over distance $\Delta x \approx q^{-1}$, the phases for all pairs of particles change significantly and the intensity of the scattered light becomes completely independent of its initial value. Thus the correlation time of the intensity fluctuations, $\tau_{c}$, is the time required for a Brownian particle to move a distance $q^{-1}$. The laws of diffusive motion stipulate that the mean square displacement of a Brownian particle over time $\Delta t$ is characterized by the relationship $\Delta x^{2}=2 D \Delta t$. Thus for $\Delta x \approx q^{-1}, \tau_{c} \approx 1 / D q^{2}$. Rigorous mathematical analysis of the process of light scattering by noninteracting small Brownian particles leads to the following expression for the normalized field correlation function in Eq. 5:

$$
\mathrm{g}^{(1)}(\tau)=\exp \left(-D q^{2} \tau\right) .
$$

\subsubsection{Polydispersity}

Equation 6 represents the simplest single exponential form of the correlation function for a solution of small, isotropic, non-interacting identical particles. In polydisperse systems, i.e., where particles of different shape or size are present in the solution, Eq. 6 has to be generalized as follows:

$$
\mathrm{g}^{(1)}(\tau)=\sum_{i} I_{i} \exp \left(-D i q^{2} \tau\right) .
$$


Here, $I_{i}$ is the normalized $\left(\Sigma I_{i}=1\right)$ contribution to scattering from particles with diffusion coefficients $D_{i}$. Reconstruction of the distribution of scattering particles over their diffusion coefficients from experimentally measured correlation function Eq. 7 is discussed in Subheading 3.3.

\subsubsection{Other Types of Dynamic Behavior}

Orientational and conformational dynamics of large (comparable to wavelength of light) particles as well as inter-particle interactions all lead to complex, non-single-exponential correlation functions, even for solutions of identical particles $(\mathbf{1 2}, 13)$. These effects are usually insignificant for scattering by particles small compared to the length of the inverse scattering vector $q^{-1}$, but become important, and often overwhelming, for larger particles. In those cases, QLS probes not the pure diffusive Brownian motion of the scatterers, but also other types of dynamic fluctuation in the solution. Fortunately, the relaxation times of these other types of fluctuations rarely depend on the scattering vector as $D q^{2}$, which is characteristic for the diffusion process. Thus, in principle, measurement of the correlation function at several different angles of scattering, and therefore at several different $q$, allows polydispersity to be distinguished from multimodal relaxation of a non-diffusive nature.

\section{The QLS System}

A QLS system consists of five elements: a source of light (laser), a sample, light collecting optics, a correlator, and the data analysis software (Fig. 2). To obtain satisfactory results, all five elements must meet certain criteria. The laser should operate in single mode and be stable. The correlator should work efficiently for sample times significantly shorter than the correlation times characteristic of the molecules under investigation. The optical setup should collect at least one photon per correlation time per coherence area. These requirements determine whether a particular system can be studied by QLS at all. If instrument performance is suitable for the study, the quality and reliability of the results will be determined by the other two elements of the QLS system, the sample and the data analysis.

QLS instruments are available commercially. Among the suppliers of QLS systems are ALV (Germany), Brookhaven Instruments (USA), Malvern Instruments (UK), and Precision Detectors (USA). We use a custom-built optical system with an Innova 90+ Argon Ion (wavelengths $488 \mathrm{~nm}$ and $514 \mathrm{~nm}$, power up to $0.5 \mathrm{~W}$ in single mode, single frequency regime) or a $\mathrm{He}-\mathrm{Ne}$ (wavelength $633 \mathrm{~nm}$ power $50 \mathrm{~mW}$ ) laser as the light source. Both lasers are from Coherent (USA). The photodetector is a Hamamatsu (Japan) R4220P PTM (photomultiplier) coupled with a 144-channel Langley-Ford (Amherst, MA) correlator and custom developed deconvolution software. Alternatively, a He-Ne laser and 


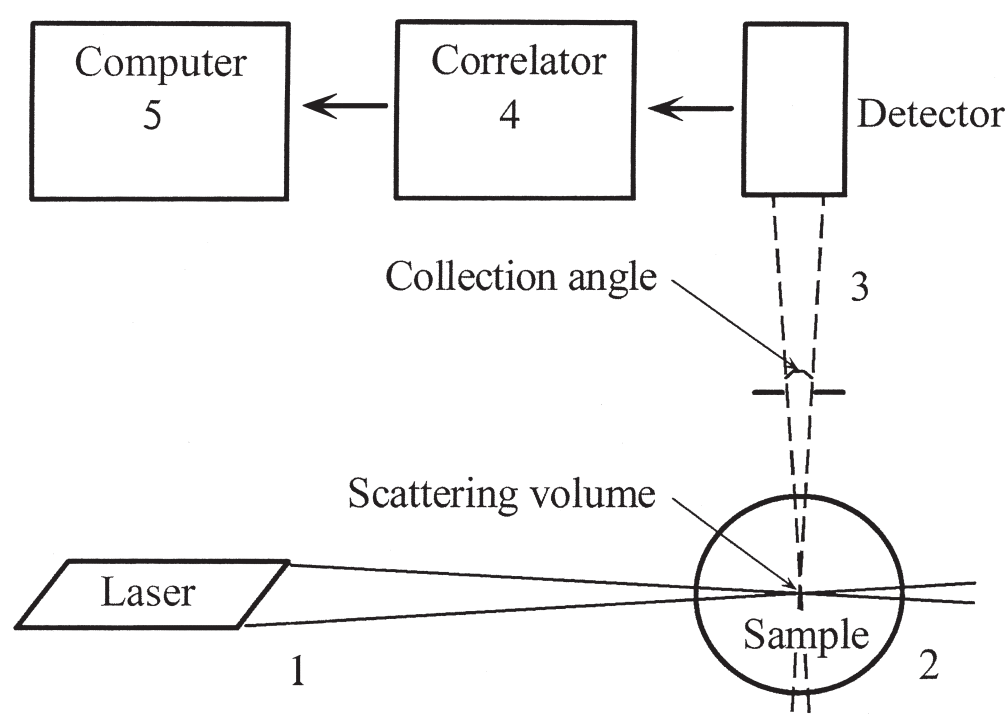

Fig 2. A block diagram of a QLS instrument. The source of light (1) consists of a laser and the focusing optics that delivers incident light into the sample. This part of the QLS system determines wavelength and intensity of the incident beam as well as the geometry of the illuminated volume. The sample cuvet (2) is the source of stray scattering both at the entry and at the exit points of the incident beam. A larger cuvet is better, but requires more sample. Collecting optics and the photodetector (3) register the intensity of the light scattered in a certain direction. Important characteristics of the collecting optics are collection volume and solid angle from which the light is collected. The intersection of the illuminated volume and the collection volume defines the scattering volume. Light intensity data obtained by the photodetector are continually fed to the correlator (4) that computes the correlation function of the intensity fluctuations. The correlation function is then analyzed (5) to determine the size and the distribution of the scattering particles.

APT (avalanche photo diode) built in the Precision Detectors 256 channel correlator is used.

\subsection{Hardware}

\subsubsection{Laser}

Intensity. The key to quality measurements in QLS is to have sufficient intensity of light scattering. If a count rate of 3 to 5 photocounts per correlation time can be achieved when light is collected from within one coherence area (see Subheading 3.1.2.), the laser intensity is sufficient. 
Single mode. The laser must operate in the single mode regime, i.e., it should generate a single transverse mode (called $\mathrm{TEM}_{00}$ ). Different transverse modes have different cross-sectional intensity profiles and very close frequencies. The mode $\mathrm{TEM}_{00}$ produces a beam with a nearly Gaussian intensity profile. If the laser generates several transverse modes simultaneously, the beam will have an irregular intensity profile. More importantly, optical "beating" between the modes would be registered by the photodetector. This may result in a distortion of the correlation function.

Stability. The fluctuations in laser intensity make the factor $I_{0}^{2}$ in Eq. 5 dependent on delay time $\tau$. From Eq. 5, it is clear that for a low efficiency factor $\gamma$, even small fluctuations in laser intensity can have significant effects on the determination of $g^{(1)}(\tau)$.

Polarization. At a given scattering angle, maximum scattering occurs in a plane perpendicular to the incident light polarization. Therefore, the incident beam should be polarized perpendicular to the plane formed by light source, sample and photodetector.

Overheating. High laser intensity may lead to increased temperature in the scattering volume. Because the diffusion coefficient is temperature-dependent, heating within the scattering volume can cause errors. Significant heating is more likely in concentrated and highly absorbing samples. To check this possibility, it is a good idea to do measurements at several intensities of the incident beam, especially when a precise absolute measurement of scattering particles size is desired.

Focusing. Generally, the narrower the beam diameter, the better. Compared to a poorly focused incident beam, a well-focused beam produces the same scattering intensity from a smaller scattering volume. This increases the coherence angle, which is an important parameter affecting the efficiency factor $\gamma$ in Eq. 5 (see Subheading 3.1.2.)

\subsubsection{Light Collection}

Scattering volume. Collecting optics ensures that light reaching the photodetector originates from (passes through) only a small "collection volume" within the sample. This minimizes stray light entering the photodetector. The intersection of the collection volume and the volume illuminated by the incident beam is termed the "scattering volume." The scattering volume does not have sharp boundaries. It is a qualitative characterization of the region within the sample where the majority of the single scattering events registered by the photodetector occur.

Particle number fluctuations. The number of particles in the scattering volume should be large. If this is not the case, intensity fluctuations associated with fluctuations in the number of particles within the scattering volume will 
contribute significantly to the measured correlation function. This contribution is dependent on the geometry of the scattering and is difficult to account for quantitatively. For this reason, a narrowly focused incident beam may not be suitable for studying large, strongly scattering objects (e.g., bacteria).

Multiple scattering. Equation 6 describes the correlation function of light that is scattered once. However, photons that are scattered several times also reach the detector. In QLS, multiple scattering should be minimized since it complicates data analysis significantly. The first scattering can only occur within the illuminated volume. The last scattering before the photon reaches the photodetector occurs within the collection volume, by definition. All intermediate scatterings can occur anywhere in the sample. Thus multiple scattering is collected from a much larger volume than is single scattering, which only comes from within the scattering volume. For this reason, multiple scattering may be a problem even in a sample with turbidity barely visible to a naked eye. To minimize effects of multiple scattering, the collection volume, the illuminated volume, and the total sample volume should be made as small as possible.

Coherence angle. Scattered light is optically collected from the scattering volume within a particular solid angle. The larger this angle, the more intensity is registered. However, if this angle becomes larger than the coherence angle, the efficiency $\gamma$ in Eq. 5 drops. The coherence angle is the angular size of the speckles in the interference pattern. The fluctuations in light intensity collected from different speckles are statistically independent. Increasing the light-collecting aperture in a QLS spectrometer beyond the coherence angle does not lead to improvement in the signal-to-noise ratio because the temporal fluctuations in the intensity are averaged out. The coherence angle is approximately $\lambda / 2 \pi L$, where $L$ is the cross-sectional size of the scattered volume perpendicular to the direction of the scattering. The coherence angle is fairly small, about $1^{\circ}$ for a $10-\mu \mathrm{m}$ wide scattering volume.

Efficiency. Parameter $\gamma$ in Eq. 5 is 1 when the scattered light is collected within the coherence angle. Otherwise the efficiency is the ratio of the coherence solid angle to the collection solid angle. If the collection angle is large compared the coherence angle, its further increase does not improve the signal-to-noise ratio in the correlation function but only magnifies the effects of instability of the incident beam. A collection angle of more then 2 to 3 coherence angles is useful only when the intensity of the scattering light falls below the intensity of stray light (see Note 2). Efficiency can be determined experimentally by examining the correlation function of a sample (see Fig. 3).

Angular dependency. Some commercial instruments allow measurements at different scattering angles. The scattering angle determines the scattering vector $\mathbf{q}$ (see Fig. 1). Equation 6 specifies how the correlation function for pure 


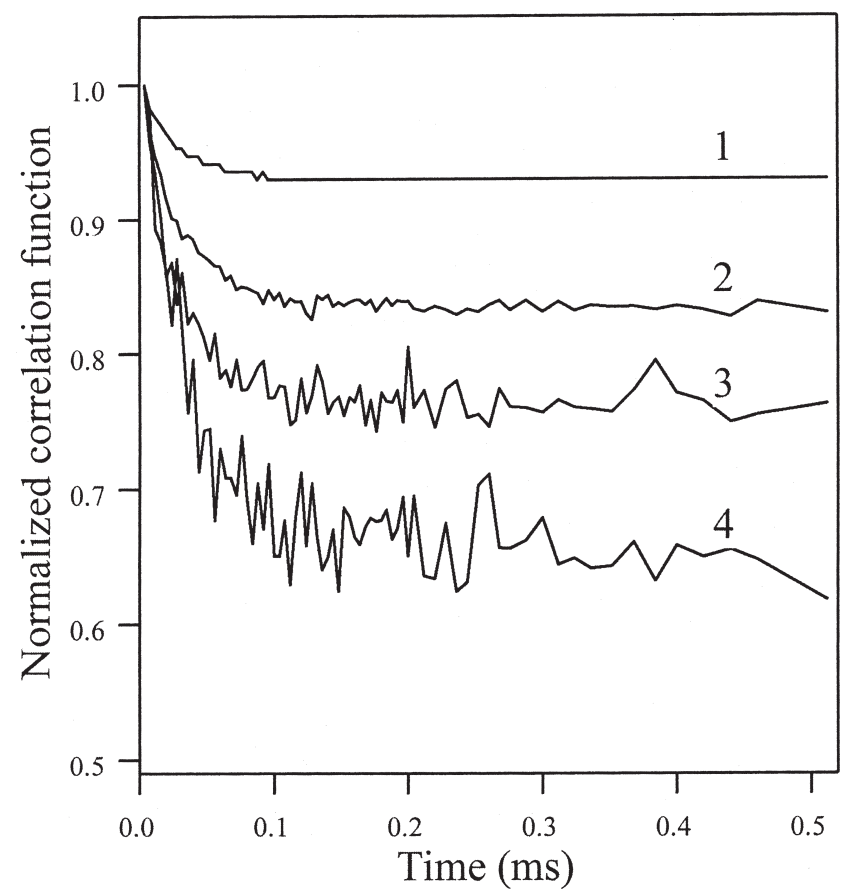

Fig 3. Correlation functions of detergent micelles (0.1\% Triton X-100) measured with different collection angles. Curves 1 to 4 represent estimated collection angles of approx 10, 3, 1, and 0.5 times the coherence solid angle, respectively. Corresponding efficiency factors $\gamma$ are $0.06,0.19,0.30$, and 0.55 . Efficiency does not reach 1 at small collection apertures because of the contribution of stray scattering into the baseline of the correlation function. Curve 2 represents the best choice of the collection angle for this sample. Note that these are short $(3 \mathrm{~s}$ each) measurements made for demonstration purposes. In practice, the measurement duration of this sample would be two orders of magnitude longer and the noise in the data would be 10 times less than in the figure.

Brownian motion depends on the absolute value of this vector. Other types of dynamic behavior (see Subheading 2.3.3.) will result in deviations in this $D q^{2}$ type dependency of the inverse relaxation time. Measurements of angular dependency thus could verify the diffusive nature of the system dynamics. Large particles, which scatter disproportionally more light, scatter even stronger at small angles (see Subheadings 2.1.2. and 2.1.3.). Unless particles $>100 \mathrm{~nm}$ are being investigated, small angle scattering should be avoided (see Note 3). In typical protein aggregation studies, there is little reason to do measurements at angles other then $90^{\circ}$. At this angle, the stray scattering from the cuvet (see Note 4) is usually at a minimum. 


\subsubsection{Correlator}

The correlator digitally computes the correlation function of the photocurrent $G^{(2)}(\tau)$. In the most common approach, the number of photons registered by the photodetector within a short time interval, the "sample time" (denoted $\Delta t$ ), is stored in the correlator. This number represents an instantaneous value of the scattered light intensity. The correlator keeps many, usually several thousands, of these consecutive intensity measurements in its memory. According to Eq. 3, to obtain the correlation function $G^{(2)}(\tau)$ at $\tau=n \Delta t$, the average product of counts separated by $n$ sample times should be determined. The number $n$ is referred to as a channel number. Clearly, the shortest delay time at which the correlation function is measured by the procedure described above is $\Delta t$ (channel 1). Modern commercial correlators can simultaneously process several hundred channels without loss of information at sample times as low as a fraction of a microsecond.

\subsubsection{Cuvet}

Where the incident beam hits the outer and inner surfaces of cuvet walls are sources of stray light that might enter the photodetector. This stray light, and the light scattered by the sample, originate from the same source and are partially coherent, producing interference patterns in addition to the interference pattern derived solely from the particles in the sample. This may result in systematic distortion of the correlation function and should be minimized. Appropriate design and good alignment of the optics, as well as careful cleaning of the cuvet, serves this goal. It is desirable to know the intensity of the background stray scattering from pure buffer and to ensure that the scattering from the sample significantly exceeds this intensity level (see Note 4).

\subsection{Sample}

\subsubsection{Role of Large Particles}

Samples monitored by QLS must be optically pure. This concept is quite different from the concept of chemical purity. Even a small weight fraction of chemically inert dust particles can completely dominate light scattering from a large population of relatively small proteins. The presence of a few large particles is often a factor in QLS experiments, especially when monitoring protein assembly. As these particles drift into and out of the scattering volume, the total scattering intensity fluctuates significantly. This can render QLS measurements unusable.

\subsubsection{Cleaning the Sample}

Avoiding dust. Dust in the air has a strong tendency to adsorb electrostatically to charged groups on the surfaces of the empty cuvet. These adsorbed 
particles will be suspended in the sample solution. If the cuvet is not to be filled completely, it is desirable to introduce the sample into the bottom of the cuvet to avoid washing dust off the cuvet walls. Do not vortex the partially filled cuvet.

Washing cuvet. Do not wash disposable cuvets or test tubes-they are usually made in a dust-free environment and filled with clean air. When washing cuvets, remember that the dust gets inside the cuvet when you empty it of washing solvent. Empty and dry cuvets in a dust-free atmosphere, e.g., in a glove box filled with filtered argon. After a cuvet is filled with sample and sealed, clean it from the outside using lens paper and lens cleaning solution or methanol.

Filtration. The easiest way to remove large impurities from solution is by filtration. Standard $0.22-\mu \mathrm{m}$ filters generally are too porous to be of use. We have found that 20-nm Anatop filters are satisfactory in most studies of protein aggregation. When aggregates of interest are too big to pass through the $20-\mathrm{nm}$ filter, they usually scatter much more than dust and do not require filtering at all.

Centrifugation is another effective way to remove large impurities from the solution, provided that the sample is spun in the same sealed cuvet in which the QLS measurements will be done. Transferring the sample into another cuvet after centrifugation defeats the purpose of the procedure. Typical airborne dust can be pelleted in $30 \mathrm{~min}$ at $5000 \mathrm{~g}$. However, there are always very "flaky" dust particles, which will not sediment by this procedure.

Flow-through filtering. Successful measurement of low molecular weight molecules is absolutely dependent on the optical purity of the sample. In studies of the amyloid $\beta$-protein $(A \beta)(14)$, we have used the intrinsic fractionation and filtering potential of size exclusion chromatography (SEC), and a continuous flow procedure for washing the QLS cuvet, to produce optically pure A $\beta$ samples. We attached one end of a micropipet to the SEC UV detector outflow line and placed the other end at the very bottom of a cuvet (a standard disposable $5 \times 50 \mathrm{~mm}, 0.5-\mathrm{mL}$, round bottom, glass test tube). In this way, "filtered" buffer is constantly washing the interior of the cuvet from the bottom up and spilling over the top. When the peak of interest is detected and fills the cuvet, the micropipet is removed from the cuvet and the cuvet is sealed and washed from the outside. This procedure, although somewhat cumbersome, provided excellent dust-free samples. We recommend it for any QLS study of peptides and small proteins.

\subsubsection{Digital Filtration}

In protein solutions, large protein aggregates may form that cannot be removed. When such aggregates pass through the scattering volume, they cause spikes of intensity, which distort the measured correlation function. Some correlators allow suppression of data acquisition during spikes of intensity. These 
algorithms are called "software dust filters" and involve establishing certain cut-off levels for the intensity of the scattered light. When software dust filtering is employed, it may be beneficial to focus the laser beam in order to minimize the scattering volume and increase the coherence angle. Dust particles or aggregates pass through a small scattering volume less frequently and in fewer numbers than through larger volumes (see discussion of laser focusing in Subheading 3.1.1.) Spikes in intensity associated with dust particles then become larger in intensity, shorter in time and less frequent. That allows for better discrimination between these spikes and the regular intensity fluctuations in the interference pattern.

\subsection{Data Analysis}

\subsubsection{Polydispersity}

The term "polydispersity" is used to describe the presence of non-identical particles in a sample. Polydispersity can be an inherent property of the sample, for instance, when polymer solutions or protein aggregation are studied, or it can be a consequence of impurities or deterioration of the sample. In the first case, the polydispersity itself is an object of interest, whereas in the second case it is an obstacle. In both instances, polydispersity significantly complicates data analysis.

For a continuous distribution of scattering particle size, Eq. 7 is generalized as follows:

$$
\mathrm{g}^{(1)}(\tau)=\frac{1}{I_{0}} \int I(D) \exp \left(-D q^{2} \tau\right) d D .
$$

Here $I(D) d D=N(D) I_{0}(D) d D$ is the intensity of light scattered by particles having their diffusion coefficient in interval $[D, D+d D], N(D) d D$ is the number of these particles in the scattering volume, and $I_{0}(D)$ is the intensity of light scattered by each of them. The goal of the mathematical analysis of QLS data is to reconstruct as precisely as possible the distribution function $I(D)$ (or $N(D)$ ) from the experimentally measured correlation function.

Unfortunately, the corresponding mathematical minimization problem is "illposed" (15), meaning that dramatically different distributions $I(D)$ lead to nearly identical correlation functions of the scattered light and therefore are equally acceptable fits to the experimental data. We discuss below three approaches for dealing with this ill-posed problem.

\subsubsection{The Direct Fit Method}

The simplest approach is the direct fit method. Here the functional form of $I(D)$ is assumed a priori (single modal, bimodal, Gaussian, etc.). The parameters of the assumed function that lead to the best fit to the experimental data 
are then determined. This method is only as good as the original guess of the functional form of $I(D)$. Moreover, the method can be misleading because it tends to "confirm" any a priori assumption made. It is also important to note that the more parameters there are in the assumed functional form of $I(D)$, the better the experimental data can be fit but the less meaningful the values of the fitting parameters become. In practice, typical QLS data allow reliable determination of about three independent parameters of the size distribution of the scattering particles.

\subsubsection{The Method of Cumulants}

The cumulant method is free from bias introduced by a priori assumptions about the shape of $I(D)$. In this approach, the focus is not on the shape of the distribution but instead on the moments of the distribution, or closely related quantities called cumulants (16). Cumulants are stable characteristics insensitive to spikes in the distribution. The first cumulant (moment) of the distribution $I(D)$, the average diffusion coefficient $\bar{D}$, can be determined from the initial slope of the field correlation function. Indeed, using Eq. 8, it is straightforward to show that:

$$
\frac{d}{d \tau} \ln \left|g^{(1)}(\tau)\right|=\frac{1}{I_{0}} \int I(D) D q^{2} d D \equiv \bar{D} q^{2}
$$

The second cumulant (moment) of the distribution can be obtained from the curvature (second derivative) of the initial part of the correlation function. As in the direct fit method, the accuracy of the real QLS experiment allows determination of at most three moments of the distribution. The first moment, $\bar{D}$, can be determined with better than $\pm 1 \%$ accuracy (see Note 5 ). The second moment, the width of the distribution, can be determined with an accuracy of \pm 5 to $10 \%$. The third moment, which characterizes the asymmetry of the distribution, usually can be estimated with an accuracy of only about $\pm 100 \%$.

\subsubsection{Regularization}

The regularization approach combines the best features of both of the previous methods. It assumes that the distribution $I(D)$ is an arbitrary, but smooth function, and seeks a non-negative distribution producing the best fit to the experimental data. The regularization requirement of smoothness precludes spikes in the distribution, allowing unique solutions to the minimization problem. There are several regularization algorithms that differ in the specific mathematical implementation of the smoothness condition. One popular program is called CONTIN (17). We have developed and use our own algorithm (18), which is also utilized in PrecisionDeconvolve software supplied with QLS instruments produced by Precision Detectors (USA). 
Smoothness parameter. The choice of the smoothness parameter is one of the most difficult and important parts of the regularization method. If the smoothing is too strong, the distribution will be very stable but will lack details. If the smoothing is too weak, false spikes can appear in the distribution. The "rule of thumb" is that the smoothing parameter should be just sufficient to provide stable, reproducible results in repetitive measurements of the same correlation function. Two facts are helpful for choosing the appropriate smoothing parameter. First, the lower the statistical errors of the measurements, the smaller the smoothing parameter can be without loss of stability. This will yield finer resolution in the reconstructed distribution $I(D)$. Second, narrow distributions generally require much less smoothing and can be reconstructed much better than can wide distributions. This is because oscillations in narrow distributions are effectively suppressed by non-negativity conditions.

Resolution of the regularization procedure. In a typical QLS experiment, regularization analysis can resolve a bimodal distribution with two narrow peaks of equal intensity if the diffusion coefficients corresponding to these peaks differ by more than a factor of approx 2.5. The moments of the distribution reconstructed by the regularization procedure with properly chosen smoothness parameter coincide closely with those obtained by the cumulant method. In Fig. 4, we present the bimodal distribution function of oligomers observed in a solution of $A \beta$ (19) computed with three different smoothing parameters. The distribution in Fig. 4A was computed without smoothing. This spiky distribution is the best fit to the measured correlation function, but it is not stable and varies from one experiment to another. The same data with an appropriately chosen smoothing parameter is shown in Fig. 4B. This distribution fits the measured data only marginally worse (about $0.5 \%$ larger average deviation) than the distribution in Fig. 4A, but is stable from one experiment to another. In Fig. 4C, we show an excessively smoothed distribution. This distribution is stable, but lacks details, and it fits the measured data noticeably worse than do the distributions in Fig. 4A,B (about 3\% larger average deviation). Note that the average diffusion coefficient and the overall width of the reconstructed distribution are affected little by the choice of smoothing parameter.

\subsection{Interpretation of Distribution of Scattering Intensity I(D)}

\subsubsection{Determination of the Sizes of Particles in Solution}

Measurement of the intensity correlation function allows evaluation of the diffusion coefficients of the scattering particles. For spherical particles, the relation between the radius $R_{h}$ and its diffusion coefficient $D$ is given by the StokesEinstein equation (20): 


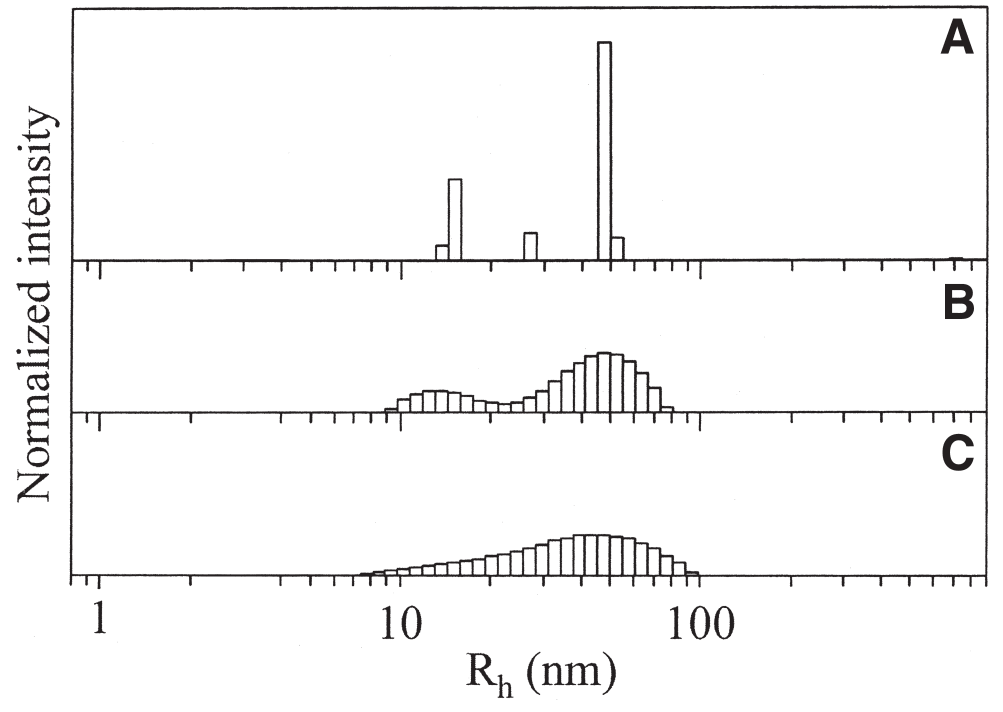

Fig 4. Oligomer size distribution of A $\beta$. (A) Distribution computed with insufficient smoothing. Peak positions in this distribution are not reliable, even though they provide the best fit to the experimental data. (B) Properly chosen regularization parameters allow observation of two fractions-oligomers with hydrodynamic radius in the range 10 to $20 \mathrm{~nm}$ and their aggregates with average radius of $60 \mathrm{~nm}$. This distribution is stable and would not vary much from one measurement to another. The average deviation from the experimental data is only $0.5 \%$ worse then in (A). (C) An excessively smoothed distribution does not show separate oligomer and aggregate populations. This distribution is stable, and it fits the experimental data well, with average deviation only $3 \%$ more than in (A), but important details are not resolved.

$$
D=\frac{k_{B} T}{6 \pi \eta R_{h}}
$$

Here $k_{B}$ is the Boltzmann constant, $T$ is the absolute temperature, and $\eta$ is solution viscosity. For non-spherical particles, Eq. 10 defines the effective hydrodynamic radius of the particle. It is customary to introduce the apparent hydrodynamic radius $R_{h}{ }^{a p p}$, defined as:

$$
R_{h}^{a p p}=\frac{k_{B} T}{6 \pi \eta D^{a p p}}
$$

where $D^{a p p}$ is the average (apparent) diffusion coefficient measured in the QLS experiment. The apparent hydrodynamic radius is calculated numerically, and in some cases analytically, for a variety of particles shapes (21) (see Note 6). 


\subsubsection{Effect of Concentration}

At finite concentrations, two additional factors affect the diffusion of particles, viscosity and inter-particle interactions. Viscosity generally increases with the concentration of macromolecular solute. According to Eq. 10, this leads to a lower diffusion coefficient and, therefore, to an increase in the apparent hydrodynamic radius. Interactions between particles can act in either direction. If the effective interaction is repulsive, which is usually the case for soluble molecules (otherwise they would not be soluble), local fluctuations in concentration tend to dissipate faster, meaning higher apparent diffusion coefficients and lower apparent hydrodynamic radii. If the interaction is attractive, fluctuations in concentration dissipate more slowly and the apparent diffusion coefficients are lower. Thus, depending on whether the effect of repulsion between particles is strong enough to overcome the effect of increased viscosity, both increasing and decreasing types of concentration dependence of the hydrodynamic radius are observed (22).

\subsubsection{Determination of the Relative Concentrations of Particles}

The regularization procedure reconstructs the distribution of the scattering intensity over diffusion coefficient, $I(D)=N(D) I_{0}(D)$, as defined by Eq. 8. Using Eq. 10, this distribution can be converted readily into a distribution over hydrodynamic radius, $R_{h}$. However, to deduce the relative concentration of particles with a particular diffusion coefficient one must know the scattering intensity from these particles, $I_{0}(D)$. As discussed in Subheading 2.1.2., the scattering intensity is proportional to the mass of an aggregate squared. The question thus reduces to the connection between mass of an aggregate and its diffusion coefficient, or equivalently, $R_{h}$. For solid spherical particles, $M \propto R_{h}^{3}$. For discs and ideal polymer coils, $M \propto R_{h}^{2}$. For long rigid rods with diameter $d$ the connection is $M \propto R_{h} \ln \left(R_{h} / d\right)$. With an appropriately postulated dependency $M\left(R_{h}\right)$, the relative molar concentration of the scattering particles is given by $N\left(R_{h}\right)=$ $I\left(R_{h}\right) / M^{2}$. The relative weight concentration is $C\left(R_{h}\right)=M N(R h)=I(R h) / M$.

In Fig. 5, we show several alternative representations of two distributions of $A \beta$ aggregates (data adapted from [23]). Panel A shows the distribution of scattering intensity over diffusion coefficient of micelle-like structures observed immediately after dissolution of of $\mathrm{A} \beta$ in $0.1 \mathrm{~N} \mathrm{HCl}$. Panel B shows the same type of distribution observed in this sample $30 \mathrm{~h}$ later, when $\mathrm{A} \beta$ fibrils were formed. These same data converted into distributions of scattering intensity over hydrodynamic radius are shown in panels $C$ and $D$, respectively. In these panels, the fraction of average $R_{h} \sim 7 \mathrm{~nm}$ represents micelles. In panel D, we also observe the contribution from A $\beta$ fibrils, which have $R_{h}$ within the 15 to $40 \mathrm{~nm}$ range in this sample. The distribution of the scattering intensity over diffusion 

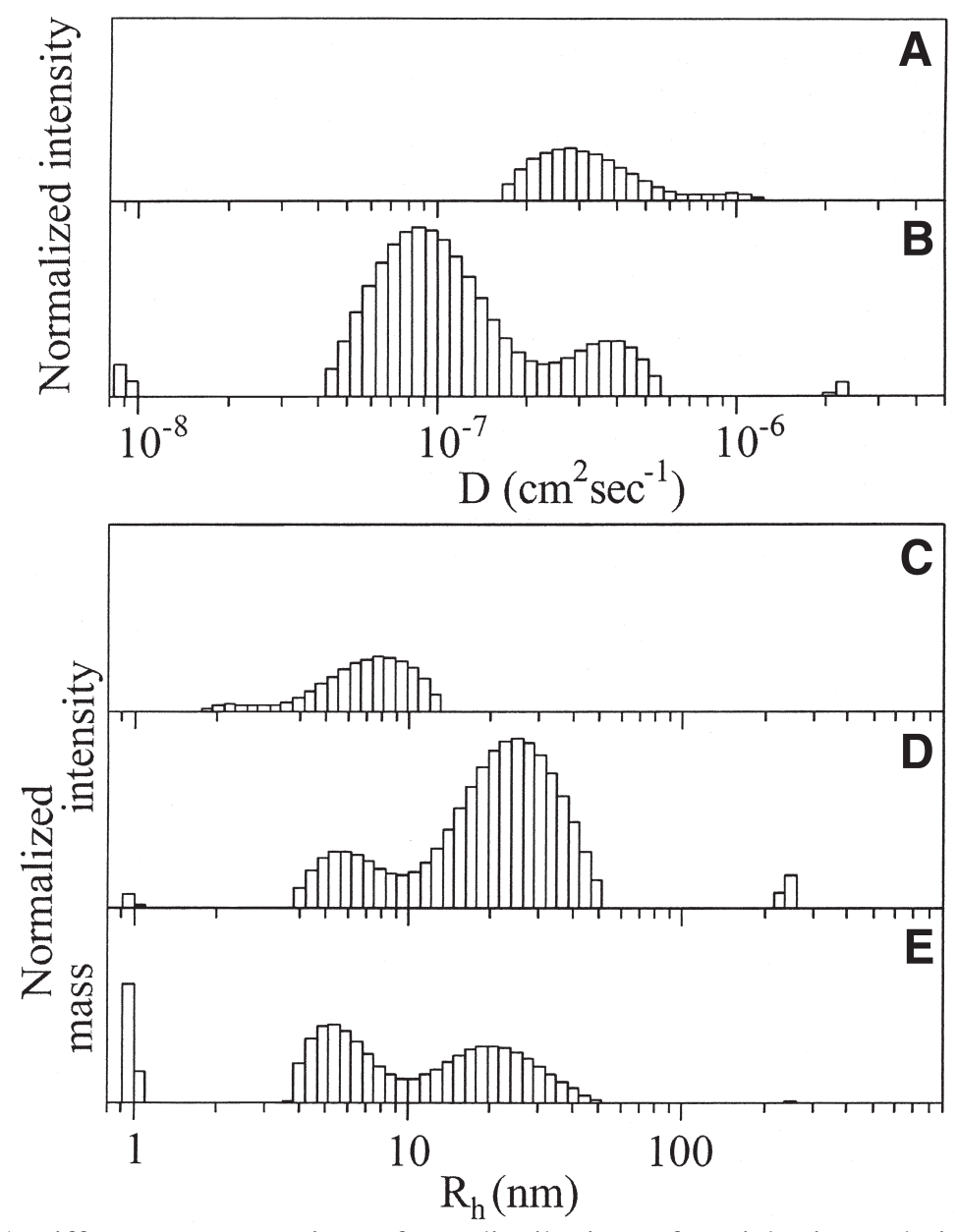

Fig. 5. Different representations of two distributions of particles in a solution of $A \beta$. (A) The distribution of scattering intensity over diffusion coefficient of micelle-like structures observed immediately after dissolution of $A \beta(1-40)$ at a concentration of $1 \mathrm{mg} / \mathrm{mL}$ in $0.1 \mathrm{~N} \mathrm{HCl}$. (B) The same distribution $30 \mathrm{~h}$ later, when $\mathrm{A} \beta$ fibrils were formed. The distributions are normalized to the total intensity of scattering, which increased approx fourfold during the incubation. Therefore, the total area of the distribution in panel B is about 4 times the area of the distribution in panel A. (C and D) The data in (A) and (B), respectively, converted into distributions of scattering intensity over hydrodynamic radius. (E) The distribution in (D) converted into the mass distribution, assuming that the mass of a scatterer is proportional to $R_{h}$.

coefficient is the primary information extracted directly from the correlation function. The average (apparent) diffusion coefficient $D^{a p p}$ is a stable characteristic and is well-reproducible. The distribution over hydrodynamic radius is 
computed using Eq. 4 and in a logarithmic scale is an appropriately shifted reflection of the distribution over the diffusion coefficient. Distribution over $R_{h}$ is a popular representation of the data, since it factors out uninteresting temperature and viscosity dependence.

Panel D in Fig. 5 shows the distribution of the intensity $I\left(R_{h}\right)=N\left(R_{h}\right) \cdot I_{0}\left(R_{h}\right)$ (see Eq. 7) coming from particles of a particular size. This distribution is renormalized in panel $\mathrm{E}$ to show the actual particle mass concentration, $N\left(R_{h}\right) \cdot M\left(R_{h}\right)$, where $M\left(R_{h}\right)$ is the mass of the particle with a given $R_{h}$. Conversion is done under assumption that the scattering intensity is proportional to $M\left(R_{h}\right) \sim R_{h}$. This assumption is an approximation roughly appropriate for fibrillar scatterers. The distribution in panel $\mathrm{E}$ indicates that about half of the material remains in micellar form after incubation, even though large fibrils dominate the scattering. Large aggregates thus may contribute significantly to scattering even though their concentration is small. The high sensitivity of QLS to large particles makes the method particularly valuable for studying aggregation processes.

\section{Notes}

1. This analysis is applicable only if the probability of multiple scattering within one particle is negligible. Analysis of scattering intensity from strongly scattering and/or absorbing particles (metallic, for example) requires a more complicated approach (24).

2. Increasing the collection aperture beyond the coherence angle increases the number of photocounts available for computation of the correlation function. That makes the correlation function look "smoother." However, it does not improve the accuracy with which the diffusion coefficient can be deduced.

3. If visible light is used, and the beam is unblocked (note that this is a safety hazard), the visual inspection of the speckle pattern observed at small angles may be informative. A bright static pattern indicates the dirt on the walls of the cuvet, whereas flashes of intensity indicate large particles crossing the beam. A pattern of speckles moving in a vertical direction is indicative of convection caused by heating.

4. Measurement of the pure buffer purified in the same way as the sample will show the level of stray scattering and the level of dust contamination. Do not use an empty cuvet to judge stray scattering. Empty cuvets produce much more scattering from inner surfaces than do filled cuvets, because of larger differences in refractive index between glass and air than between glass and water.

5. The average diffusion coefficient $\bar{D}$ is a stable characteristic of the distribution. That is not so for the average size of the particles (hydrodynamic radius defined in Subheading 3.4.1.) $\bar{D}$ is proportional to $\overline{1 / R_{h}}$, which is not the same as $1 / \bar{R}_{h}$. As a consequence, an average hydrodynamic radius $\bar{R}_{h}$ for a wide distribution may vary dramatically from one measurement to another. A much better characteristic of the typical particle size is $R_{h}^{a p p}$ computed from $D^{a p p}$ using Eq. 11. 
6. For non-spherical particles, the diffusion coefficient is actually a tensor-the rate of particle diffusion in a certain direction depends on the particle orientation relative to this direction. Small particles, as they diffuse over a distance $q^{-1}$, change their orientation many times. QLS measures the average (apparent) diffusion coefficient for these particles. Particles of a size comparable to, or larger than, $q^{-1}$ essentially preserve their orientation as they travel a distance smaller than their size. For these particles, the single exponential expression of Eq. 6 for the field correlation function is not strictly applicable. This fact is particularly important in QLS applications designed for studying long fibrils (8).

\section{Acknowledgments}

This work was supported by the National Institutes of Health Grants AG14366, AG18921, NS38328 (to D.B.T.), and IY05127 (to G.B.B), by the Foundation for Neurological Diseases (to D.B.T), and by the National Aeronautics and Space Administration Grant NAG8-1659 (to G.B.B.).

\section{References}

1. Pecora, R. (1985) Dynamic Light Scattering: Applications of Photon Correlation Spectroscopy. Plenum Press, New York.

2. Schmitz, K. S. (1990) An Introduction to Dynamic Light Scattering by Macromolecules. Academic Press, Boston.

3. Selkoe, D. J. (1994) Alzheimer's disease-A central role for amyloid. J. Neuropath. Exp. Neurol. 53, 438-447.

4. Prusiner, S. B. (1998) Prions. Proc. Natl. Acad. Sci. USA 95, 13363-13383.

5. Kirkitadze, M. D., Bitan, G., and Teplow, D. B. (2002) Paradigm shifts in Alzheimer's disease and other neurodegenerative disorders: the emerging role of oligomeric assemblies. J. Neurosci. Res. 69, 567-577.

6. Eaton, W. A. and Hofrichter, J. (1990) Sickle cell hemoglobin polymerization. Adv. Prot. Chem. 40, 63-279.

7. Benedek, G. B. (1997) Cataract as a protein condensation disease. Invest. Ophtalmol. Vis. Sci. 38, 1911-1921.

8. Lomakin, A., Benedek, G. B., and Teplow, D. B. (1999) Monitoring protein assembly using quasielastic light scattering spectroscopy. Meth. Enzymol. 309, 429-459.

9. Debye, P. (1947) Molecular-weight determination by light scattering. J. Phys. Col. Chem. 51, 18-32.

10. Benedek, G. B. (1971) Theory of transparency of eye. Appl. Optics. 10, 459-473.

11. Kerker, M. (1969) The scattering of light and other electromagnetic radiation. Academic Press, New York.

12. Chichoki, B. and Felderhof, B. U. (1993) Dynamic scattering function of a semidiluted suspension of hard spheres. J. Chem. Phys. 98, 8186-8193.

13. Balabonov, S. M., Ivanova, M. A., Klenin, S. I., Lomakin, A., Molotkov, V. A., and Noskin, V. A. (1988) Quasielastic light scattering study of linear macromolecules dynamics. Macromolecules 21, 2528-2535. 
14. Walsh, D. M., Lomakin, A., Benedek, G. B., Condron, M. M., and Teplow, D. B. (1997) Amyloid $\beta$-protein fibrillogenesis. Detection of protofibrillar intermediate. J. Biol. Chem. 272, 22364-22372.

15. Tikhonov, A. N. and Arsenin, V. Y. (1977) Solution of Ill-Posed Problems. Halsted Press, Washington.

16. Koppel, D. E. (1972) Analysis of macromolecular polydispersity in intensity correlation spectroscopy. The method of cumulants. J. Chem. Phys. 57, 4814-4820.

17. Provencher, S. W. (1982) A constrained regularization method for inverting data represented by linear algebraic or integral equations. Comput. Phys. Commun. 27, 213-227.

18. Braginskaya, T. G., Dobitchin, P. D., Ivanova, M. A., et al. (1983) Analysis of the polydispersity by photon correlation spectroscopy: regularization procedure. Physica Scripta 28, 73-79.

19. Bitan, G., Kirkitadze, M. D., Lomakin, A., Vollers, S. S., Benedek, G. B., and Teplow, D. B. (2003) Amyloid $\beta$-protein (A $\beta$ ) assembly: $A \beta 40$ and A $\beta 42$ oligomerize through distinct pathways. Proc. Natl. Acad. Sci. USA 100, 330-335.

20. Einsten, A. (1905) Über die von der molekularkinetischen Theorie der Wärme geforderte Bewegung von in ruhenden Flüssigkeiten suspendierten Teilchen. Annalen der Physik und Chemie 17, 549-560.

21. de la Torre, J. G. and Bloomfield, V. A. (1981) Hydrodynamic properties of complex, rigid, biological macromolecules: theory and applications. Quarterly Reviews of Biophysics 14, 81-139.

22. Muschol, M. and Rosenberger, F. (1995) Interactions in undersaturated and supersaturated lysozyme solutions: static and dynamic light scattering results. J. Chem. Phys. 103, 10424-10432.

23. Yong, W., Lomakin, A., Kirkitadze, M. D., Teplow, D. B., Chen, S.-H., and Benedek, G. B. (2001) Structure determination of micelle-like intermediates in amyloid $\beta$-protein assembly by using small angle neutron scattering. Proc. Natl. Acad. Sci. USA 99, 150-154.

24. Van de Hulst, H. C. (1981) Light Scattering by Small Particles. Dover, New York. 